\title{
Sur une équation aux valeurs propres de vibration-rotation pour tout potentiel électronique d'une molécule diatomique
}

\author{
H. Kobeissi \\ Faculté des Sciences-I, Université Libanaise, Hadath-Beyrouth, Liban \\ et Groupe de Physique Moléculaire et Atomique, au Conseil National de la Recherche Scientifique
}

(Reçu le 20 août 1980, révisé le 23 décembre, accepté le 17 février 1981)

\begin{abstract}
Résumé. - Une «équation aux valeurs propres " pour l'équation radiale de Schroedinger est proposée. L'équation est valable pour tout potentiel électronique d'une molécule diatomique ; le potentiel pouvant être donné par une fonction analytique ou sous une forme numérique. Une application numérique est donnée pour le potentiel de Morse de l'état fondamental de la molécule $\mathrm{Li}_{2}$, ainsi que pour un potentiel modèle d'une méthode conventionnelle. Notre méthode permet de calculer le niveau d'énergie $E_{v}$ et la constante de rotation $B_{v}$ avec une précision égale à celle de l'ordinateur utilisé.
\end{abstract}

Abstract. - An « eigenvalue equation » is given for the radial Schroedinger equation for any electronic potential of a diatomic molecule. The potential function may be given by an analytic or a numerical function. A numerical application to the Morse potential of the $\mathrm{X} \sum-\mathrm{Li}_{2}$ and to a model potential of a conventional method is presented. The calculated eigenvalues agree with the theoretical ones up to the 9 th significant figure.

1. Introduction. - Dans l'approximation de BornOppenheimer [1], une molécule diatomique, dans un état électronique donné, est régie par l'équation radiale de Schroedinger [2] :

$$
\frac{\mathrm{d}^{2} y(x)}{\mathrm{d} x^{2}}=f(x) y(x)
$$

où

$$
f(x)=-\frac{2 \mu}{\hbar^{2}}\left[E_{v J}-U(x)\right]+\frac{J(J+1)}{r^{2}}
$$

$U(x)=$ potentiel de l'état électronique,

$E_{v J}=$ niveau d'énergie de vibration-rotation,

$x=r-r_{\mathrm{e}}$; où $r$ est la distance internucléaire, $r_{\mathrm{e}}$ étant

la valeur de $r$ à l'équilibre,

$v$ et $J$ sont les nombres quantiques de vibration et de rotation.

La solution de cette équation représente la fonction d'onde de vibration-rotation $\psi_{v \delta}(x)$ quand elle obéit aux conditions suivantes [2] :

i) $\psi$ et $\mathrm{d} \psi / \mathrm{d} x$ sont définies et continues en tout point.

ii) $\psi$ obéit aux conditions aux limites

$$
\begin{aligned}
\psi(x) & \rightarrow 0 \\
x & \rightarrow+\infty \\
x & \rightarrow-r_{\mathrm{e}} .
\end{aligned}
$$

En dehors de quelques cas particuliers où l'on connaît les valeurs propres $E_{v J}$ de l'équation (1) (potentiel de Dunham [3], de Morse [4]), la détermination de la valeur propre est associée à celle de la fonction propre $\psi_{v J}$ par une méthode itérative basée sur le travail de Löwdin [5], developpée essentiellement par Cooley [6], Zare [7] et Cashion [8] et reprise par d'autres méthodes équivalentes $[9,10]$.

Le but de ce travail est de dissocier la détermination de la valeur propre de celle de la fonction propre, en donnant une "équation aux valeurs propres" indépendante de la fonction propre $\psi_{v j}$. Cette équation est écrite grâce à l'introduction des fonctions canoniques de vibration-rotation.

2. Les fonctions canoniques de rotation-vibration. Dans un travail récent [11], nous avons étendu la définition des fonctions canoniques de vibration [12, 13] au cas de la vibration-rotation. Ces fonctions $\alpha_{v J}(x)$ et $\beta_{v J}(x)$ sont définies par :

$$
\begin{aligned}
& \alpha_{v J}(x)=1+\int_{v}^{x} R(x, t) \mathrm{d} t \\
& \beta_{v J}(x)=x+\int_{v}^{x} t R(x, t) \mathrm{d} t
\end{aligned}
$$


où le « noyau résolvant » $R(x, t)$ est défini par

$$
\begin{aligned}
R(x, t) & =\sum_{i=0}^{\infty} K_{i}(x, t) \\
K_{i}(x, t) & =\int_{t}^{x} K_{0}(x, z) K_{i-1}(z, t) \mathrm{d} z ; i \geqslant 1 \\
K_{0}(x, t) & =(x-t) f(t) .
\end{aligned}
$$

Nous avons montré [12] que ces fonctions canoniques sont des solutions de l'équation (1) ayant pour valeurs initiales

$$
\begin{array}{ll}
\alpha_{v J}(0)=1, & \alpha_{v J}^{\prime}(0)=0, \\
\beta_{v J}(0)=0, & \beta_{v J}^{\prime}(0)=1,
\end{array}
$$

et qu'elles sont liées à la fonction d'onde par la relation

$$
\psi_{v J}(x)=\psi_{v J}(0) \alpha_{v J}(x)+\psi_{v J}^{\prime}(0) \beta_{v J}(x) .
$$

Les fonctions canoniques présentent sur la fonction d'onde deux avantages :

i) leur calcul ne pose pas un problème de « valeurs initiales "),

ii) les valeurs initiales de la fonction d'onde se déduisent des fonctions canoniques.

En effet, les conditions aux limites (2) imposées à la fonction $\psi_{v J}$ entraînent

$$
\begin{aligned}
\frac{\psi_{v J}^{\prime}(0)}{\psi_{v J}(0)} & =\lim _{x \rightarrow+\infty}-\frac{\alpha_{v J}(x)}{\beta_{v J}(x)} \\
& =\lim _{x \rightarrow-r_{e}}-\frac{\alpha_{v J}(x)}{\beta_{v J}(x)}
\end{aligned}
$$

3. L'équation aux valeurs propres. - En calculant les fonctions canoniques $\alpha$ et $\beta$ à partir des expressions (3) et (4) et pour une valeur quelconque du «paramètre " $E$, on peut déterminer les deux quantités suivantes :

$$
\begin{aligned}
& p^{+}(E)=\lim _{x \rightarrow+\infty}-\frac{\alpha(x)}{\beta(x)} \\
& p^{-}(E)=\lim _{x \rightarrow-r_{e}}-\frac{\alpha(x)}{\beta(x)}
\end{aligned}
$$

En considérant, d'autre part, la fonction

$$
z(x)=\beta(x) / \alpha(x),
$$

on peut montrer que :

$$
z^{\prime}(x)=\frac{\alpha \beta^{\prime}-\alpha^{\prime} \beta}{\alpha^{2}}=\frac{1}{\alpha^{2}} .
$$

On sait que la fonction $\alpha$ oscille entre les deux points de retour $x_{v}^{+}$et $x_{v}^{-}$et augmente indéfiniment au-delà des points de retour [12]. On peut écrire pour le demiaxe positif :

$$
\lim _{x \rightarrow+\infty} z(x)=z\left(x_{v}^{+}\right)+\int_{x_{v}^{+}}^{+\infty} \frac{\mathrm{d} t}{\alpha^{2}}=-\frac{1}{p^{+}(E)}
$$

et pour le demi-axe négatif :

$$
\lim _{x \rightarrow-r_{\mathrm{e}}} z(x)=z\left(x_{v}^{-}\right)+\int_{x_{v^{-}}}^{-r_{\mathrm{e}}} \frac{\mathrm{d} t}{\alpha^{2}}=-\frac{1}{p^{-}(E)} .
$$

La condition (5) qui impose la continuité de la fonction d'onde en $x=0$ devient

$$
\frac{\beta\left(x_{v}^{+}\right)}{\alpha\left(x_{v}^{+}\right)}+\int_{x_{v}^{+}}^{+\infty} \frac{\mathrm{d} t}{\alpha^{2}}=\frac{\beta\left(x_{v}^{-}\right)}{\alpha\left(x_{v}^{-}\right)}+\int_{x_{\bar{v}}^{-}}^{-r_{\mathrm{e}}} \frac{\mathrm{d} t}{\alpha^{2}} .
$$

Cette équation est l'équation aux valeurs propres, puisqu'elle n'est vérifiée que lorsque $E=E_{v J}$. Elle s'applique à tout potentiel électronique d'une molécule diatomique sans aucune restriction à la forme (analytique ou "numérique ») de la fonction potentielle $U(x)$.

Cette équation dépend de $E$ et non des valeurs initiales $\psi(0)$ et $\psi^{\prime}(0)$, de la fonction d'onde. Elle présente donc pour la résolution de l'équation radiale de Schroedinger, l'avantage de dissocier la détermination de la valeur propre $E_{v J}$ de celle de la fonction propre $\psi_{v J}$

4. Application numérique. - Nous présentons, à titre d'exemple, une application numérique au potentiel de Morse pour l'état fondamental de la molécule

Tableau I. - Valeurs propres de vibration pour les 15 premiers niveaux du potentiel de Morse de l'état $\mathrm{X} \sum-\mathrm{Li}_{2}$. (Valeurs calculées comparées aux valeurs théoriques; en $\mathrm{cm}^{-1}$.)

[Eigenvalues for the first 15 vibrational levels of the Morse potential of the $\mathrm{X} \sum-\mathrm{Li}_{2}$ state. (The calculated values compared to the theoretical values; in $\mathrm{cm}^{-1}$.)]

\begin{tabular}{rcc|c}
$v$ & \multicolumn{1}{c}{$E_{v}^{\mathrm{t}}$} & $E_{v}^{\mathrm{c}}$ & $\frac{E_{v}^{\mathrm{c}}-E_{v}^{\mathrm{t}}}{E_{v}^{\mathrm{t}}} \mid \times 10^{8}$ \\
- & - & - & - \\
0 & 175,0625 & 175,06250003 & 0,02 \\
1 & 521,2725 & 521,27249844 & 0,30 \\
2 & 862,2625 & 862,26248400 & 1,97 \\
3 & 1198,0325 & 1192,03248400 & 1,35 \\
4 & 1528,5825 & 1528,58248400 & 1,05 \\
5 & 1853,9125 & 1853,91249170 & 0,44 \\
6 & 2174,0225 & 2174,02248750 & 0,58 \\
7 & 2488,9125 & 2488,91249650 & 0,14 \\
8 & 2798,5825 & 2798,58248100 & 0,68 \\
9 & 3103,0325 & 3103,03249650 & 0,11 \\
10 & 3402,2625 & 3402,26249890 & 0,04 \\
11 & 3696,2725 & 3696,27248120 & 0,50 \\
12 & 3985,0625 & 3985,06250200 & 0,05 \\
13 & 4268,6325 & 4268,63248600 & 0,33 \\
14 & 4546,9825 & 4546,98249290 & 0,16
\end{tabular}


$\mathrm{Li}_{2}$. La fonction potentielle de Morse [4] présente, à cet effet, l'avantage de connaître la valeur théorique $E_{v J}^{t}$ de la valeur propre donnée par la relation :

$$
\begin{aligned}
E_{v J}^{\mathrm{t}}= & W_{\mathrm{e}}\left(v+\frac{1}{2}\right)-W_{\mathrm{e}} x_{\mathrm{e}}\left(v+\frac{1}{2}\right)^{2}+ \\
& +J(J+1) B_{v}+J^{2}(J+1)^{2} D_{v}+\cdots .
\end{aligned}
$$

Les constantes utilisées sont celles données par Kusch et Hessel [14].

Nous donnons les résultats dans le tableau I pour $v$ allant de 0 à 14 et pour $J=0$. Nous remarquons que les valeurs calculées $E_{v}^{\mathrm{c}}\left(2^{\mathrm{e}}\right.$ colonne) sont égales aux valeurs théoriques correspondantes à quelques millionièmes de $\mathrm{cm}^{-1}$ près. L'écart relatif moyen entre valeur calculée et valeur théorique pour les 15 niveaux considérés est de $5 \times 10^{-9}$. Cette imprécision correspond à celle de l'ordinateur utilisé (Texas Instruments $980 \mathrm{~A}$ ).

5. Discussion. - Afin de comparer la valeur propre $E_{v J}$ calculée par notre méthode à celle calculée par une méthode conventionnelle et éprouvée, nous avons choisi de reprendre le potentiel modèle déjà utilisé par Cashion [8] et donné par la fonction :

$$
U(x)=D\left[1-\mathrm{e}^{-a x}\right]^{2}-D .
$$

Nous donnons dans le tableau II nos résultats $E_{v}^{\mathbf{K}}$ comparés à ceux de Cashion $E_{v}^{\mathrm{C}}$ et aux valeurs théoriques $E_{v}^{\mathbf{t}}$, et ceci pour les 6 premiers niveaux de vibration publiés par Cashion. Dans le tableau III nous donnons les constantes de rotation $B_{v}^{\mathbf{K}}$ calculées par notre méthode, comparées à celles de Cashion $B_{v}^{\mathrm{C}}$ et aux valeurs théoriques $B_{v}^{\mathrm{t}}$. (Nous avons déduit $B_{v}$ de l'équation (7) en donnant à $J$ deux valeurs différentes et à $D_{v}$ la valeur théorique donnée par Cashion.)

Nous soumettons enfin notre méthode à un troisième test : nous comparons la dérivée logarithmique $\psi^{\prime}(0) / \psi(0)$ déduite de l'équation (6) quand celle-ci

Tableau II. - Valeurs propres de vibration pour les 6 premiers niveaux du potentiel modèle de Cashion [8]. (Valeurs calculées comparées aux valeurs théoriques et à celles de Cashion; en $\mathrm{cm}^{-1}$.)

[Eigenvalues for the first 6 vibrational levels of the Cashion potential [8]. (The calculated values compared to the theoretical values and to those of Cashion; in $\mathrm{cm}^{-1}$.)]

Tableau III. - Constantes de rotation pour les 6 premiers niveaux de vibration du potentiel modèle de Cashion [8]. (Valeurs calculées comparées aux valeurs théoriques et à celles de Cashion; en $\mathrm{cm}^{-1}$.)

[Rotation constants for the first 6 vibrational levels of the Cashion potential [8]. (The calculated values

\begin{tabular}{|c|c|c|c|}
\hline$v$ & $B_{v}^{\mathrm{t}}$ & $B_{v}^{C}$ & $B_{v}^{\mathrm{K}}$ \\
\hline & - & - & - \\
\hline 0 & 0,16981808 & 0,169812 & 0,16981808 \\
\hline 1 & 0,16470616 & 0,164698 & 0,16470589 \\
\hline 2 & 0,15954333 & 0,159532 & 0,15954093 \\
\hline 3 & 0,15432959 & 0,154300 & 0,15431649 \\
\hline 4 & 0,14906495 & 0,149029 & 0,14903360 \\
\hline 5 & 0,14374940 & 0,143687 & 0,1436909 \\
\hline
\end{tabular}
are compared to the theoretical values and to those of Cashion; in $\mathrm{cm}^{-1}$.)]

est vérifiée, à la valeur théorique donnée par la fonction de Morse [15]. Nous donnons dans le tableau IV la valeur calculée $p_{v}^{\mathrm{c}}$ et la valeur théorique $p_{v}^{\mathrm{t}}$ de $\psi^{\prime}(0) / \psi(0)$ pour les mêmes niveaux de vibration considérés du potentiel modèle de Cashion.

Tableau IV. - Dérivée logarithmique de la fonction d'onde $p_{v}=\psi_{v}^{\prime}(0) / \psi_{v}(0)$ à l'origine pour les 6 premiers niveaux de vibration du potentiel de Cashion [8]. (Valeurs calculées $p_{v}^{\mathrm{c}}$ comparées aux valeurs théoriques $p_{v}^{i}$ )

[Logarithmic derivative $p_{v}=\psi_{v}^{\prime}(0) / \psi_{v}(0)$ of the wavefunction at the origin for the first 6 vibrational levels of the Cashion potential [8]. (The calculated values are compared to the theoretical values.)]

\begin{tabular}{ccr}
$v$ & $p_{v}^{\mathrm{t}}$ & \multicolumn{1}{c}{$p_{v}^{\mathrm{c}}$} \\
$\overline{0}$ & $\overline{-}$ & $\overline{-494} 439$ \\
1 & 0,494439 & $-23,124790$ \\
2 & $-23,124794$ & 10,290561 \\
3 & 10,290562 & $-4,159660$ \\
4 & $-4,159658$ & $-54,150713$ \\
5 & $-54,150703$ & 19,505025
\end{tabular}

L'étude des trois derniers tableaux montre que : - l'écart moyen $\Delta E_{v}$ entre valeur calculée et valeur théorique du niveau d'énergie $E_{v}$ pour les niveaux considérés est de $135 \times 10^{-6} \mathrm{~cm}^{-1}$ pour les résultats de Cashion ; il n'est que de $0,5 \times 10^{-6} \mathrm{~cm}^{-1}$ pour nos résultats ;

- l'écart moyen $\Delta B_{v}$ de la constante de rotation est de $4,3 \times 10^{-6} \mathrm{~cm}^{-1}$ pour Cashion et de $2,9 \times 10^{-6} \mathrm{~cm}^{-1}$ pour nos résultats. Cette imprécision correspond au $9^{\mathrm{e}}$ chiffre significatif de la valeur propre $E_{v \mathrm{~J}}$, c'est-à-dire à la limite de la précision de l'ordinateur utilisé ;

- l'écart relatif moyen $|\Delta p / p|$ de la pente de la fonction d'onde à l'origine est de 1,6 × 10 ${ }^{-7}$.

Ces résultats confirment la validité de la méthode. 
6. Conclusion. - Nous avons présenté une « équation aux valeurs propres " pour un potentiel électronique d'une molécule diatomique. Cette équation permet de dissocier, dans la résolution de l'équation radiale de Schroedinger, la détermination du niveau d'énergie de celle de la fonction d'onde.

Cette équation s'écrit pour la vibration-rotation aussi bien-que pour la vibration pure. Elle permet donc de déterminer les niveaux d'énergie de vibration aussi bien que les constantes de rotation et les cons- tantes de distorsion. Elle s'applique à toute fonction potentielle de tout état électronique de n'importe quelle molécule diatomique.

Remerciements. - Les calculs ont été effectués au Centre de Calcul Scientifique du Conseil National de la Recherche Scientifique au Liban, nous tenons à remercier les responsables de ce centre pour leur aimable collaboration.

\section{Bibliographie}

[1] Born, M. et Oppenheimer, R., Ann. Phys. 84 (1927) 457.

[2] Herzberg, G., Spectra of Diatomic Molecules (D. Van Nostrand (Canada) Ltd., Toronto) 1950

[3] Dunham, J. L., Phys. Rev. 41 (1932) 721.

[4] Morse, P., Phys. Rev. 34 (1929) 57.

[5] LöWdIN, P. O., J. Mol. Spectrosc. 10 (1963) 12.

[6] CoOley, J. W., Math. Computation 15 (1961) 363.

[7] Zare, R. N., J. Chem. Phys. 40 (1964) 1934.

[8] Cashion, J. K., J. Chem. Phys. 39 (1963) 1872.

[9] Kobeissi, H., Thèse, Lyon (1972).
[10] HajJ, F. H., Kobeissi, H., Nassif, N., J. Comp. Phys. 16 (1974) 150.

[11] Kobeissi, H. et DAGHeR, M., IJQC Proceedings of the International Symposium on Atomic, Molecular and Solid State Theory (1979).

[12] Kobeissi, H. et Tergiman, Y. S., J. Physique 35 (1974) 635.

[13] Kobeissi, H. et Alameddine, M. A., J. Physique 39 (1978) 43.

[14] Kusch, P. et Hessel, M. M., J. Chem. Phys. 67 (1977) 586.

[15] Kobeissi, H. et Grandmontagne, R., C. R. Hebd. Séan. Acad. Sci. 271 (1970) 638. 